\title{
Perianal Metastasis of Small Cell Lung Carcinoma: A Case Report
}

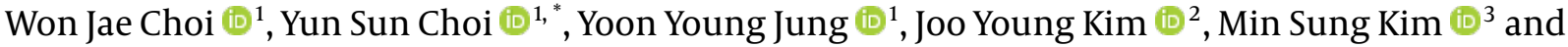 \\ Jin Woo Kim (iD 4 \\ ${ }^{1}$ Department of Radiology, Nowon Eulji Medical Center, Eulji University, Seoul, South Korea \\ ${ }^{2}$ Department of Pathology, Nowon Eulji Medical Center, Eulji University, Seoul, South Korea \\ ${ }^{3}$ Department of Surgery, Nowon Eulji Medical Center, Eulji University, Seoul, South Korea \\ ${ }^{4}$ Department of Orthopedic Surgery, Nowon Eulji Medical Center, Eulji University, Seoul, South Korea \\ "Corresponding author: Department of Radiology, Nowon Eulji Medical Center, Eulji University, Seoul, South Korea. Email: cys0128@eulji.ac.kr
}

Received 2020 November 08; Revised 2020 December 02; Accepted 2021 March 15.

\begin{abstract}
The most common sites for metastasis of lung cancer are the bones, liver, adrenal glands, and brain. Only a few studies have reported on perianal or perirectal metastasis of lung cancer. Herein, we report the case of a 78-year-old man who developed distant perianal metastasis of small cell lung carcinoma (SCLC), presenting as a palpable mass and pain in the buttock, diagnosed through magnetic resonance imaging. Immunohistochemical staining confirmed perianal metastasis from SCLC. It is important to be aware of this uncommon manifestation of lung cancer, which will help in early diagnosis and proper treatment.
\end{abstract}

Keywords: Small Cell Lung Carcinoma, Metastasis, Perianal, Magnetic Resonance Imaging

\section{Introduction}

Primary lung cancer is the most frequent malignancy with bones, liver, adrenal glands, and brain as typical metastatic sites (1-4). Perianal/anal or perirectal/rectal metastasis of lung cancer is extremely rare, with only a few cases reported in the literature (1-8). The radiological features of gastrointestinal metastases, including the perirectoanal region, have not been fully investigated $(1,2)$. Herein, we report a case of perianal metastasis of small cell lung carcinoma (SCLC) with its imaging features.

\section{Case Presentation}

A 78-year-old man, who was a former smoker, visited the surgical outpatient department of our hospital with anal pain. The patient was diagnosed as case of a limited-stage SCLC (T4N1M0) on presentation and received chemotherapy and radiation therapy. Physical examination revealed a tiny anal polyp with an internal hemorrhoid. Routine laboratory test results were normal. The patient then visited the orthopedic surgery outpatient department with complaints of pain and a palpable mass in the right buttock area. Two months later, the patient showed no improvement in symptoms and was examined further. Magnetic resonance imaging (MRI) of the pelvis showed a $5.2 \times 7.3 \times 5.6 \mathrm{~cm}$ mass in the right perianal area. The mass showed intermediate signal intensity on
T1-weighted imaging, with high signal intensity (suggesting necrosis) at the center and peripheral intermediate signal intensity on fat suppressed T2-weighted imaging (Figure $1 \mathrm{~A}$ and $\mathrm{B}$ ). Invasion of the right anal sphincter and puborectal muscles was suspected (Figure 1C). Thickening of the surrounding skin and superficial subcutaneous layer with an enlarged right inguinal lymph node $(1.2 \times 1.8 \times 1.3$ $\mathrm{cm}$ ) was observed (Figure 1B). After performing MRI, we suspected the possibility of metastatic lung cancer in the perianal mass and suggested soft tissue sarcoma as a differential diagnosis. A contrast-enhanced computed tomography (CT) scan of the pelvis was performed for palliative radiation therapy planning. It showed an irregularly shaped enhancing mass in the right perirectoanal area(Figure 1D).

The patient underwent ultrasound-guided biopsy of the perianal mass with an 18-gauge core needle for histological confirmation. Histologically, the specimen was composed of nests of tumor cells with a hyalinized stroma (Figure 2A). The tumor was composed of small round cells with finely dispersed chromatin, nuclear molding, mitosis, and necrosis (Figure 2B). The tumor cells were diffusely positive for CD56 and cytokeratin on immunohistochemistry, implying metastasis from SCLC (Figure 2C and D) due to the histological and immunohistochemical features identical to those of lung cancer (image not shown).

The palliative radiotherapy on the perianal area was performed with a daily dose of $250 \mathrm{cGy}$ for 1 month, and a total dose of 5500 cGy. On follow-up after 1 year, pelvis CT 

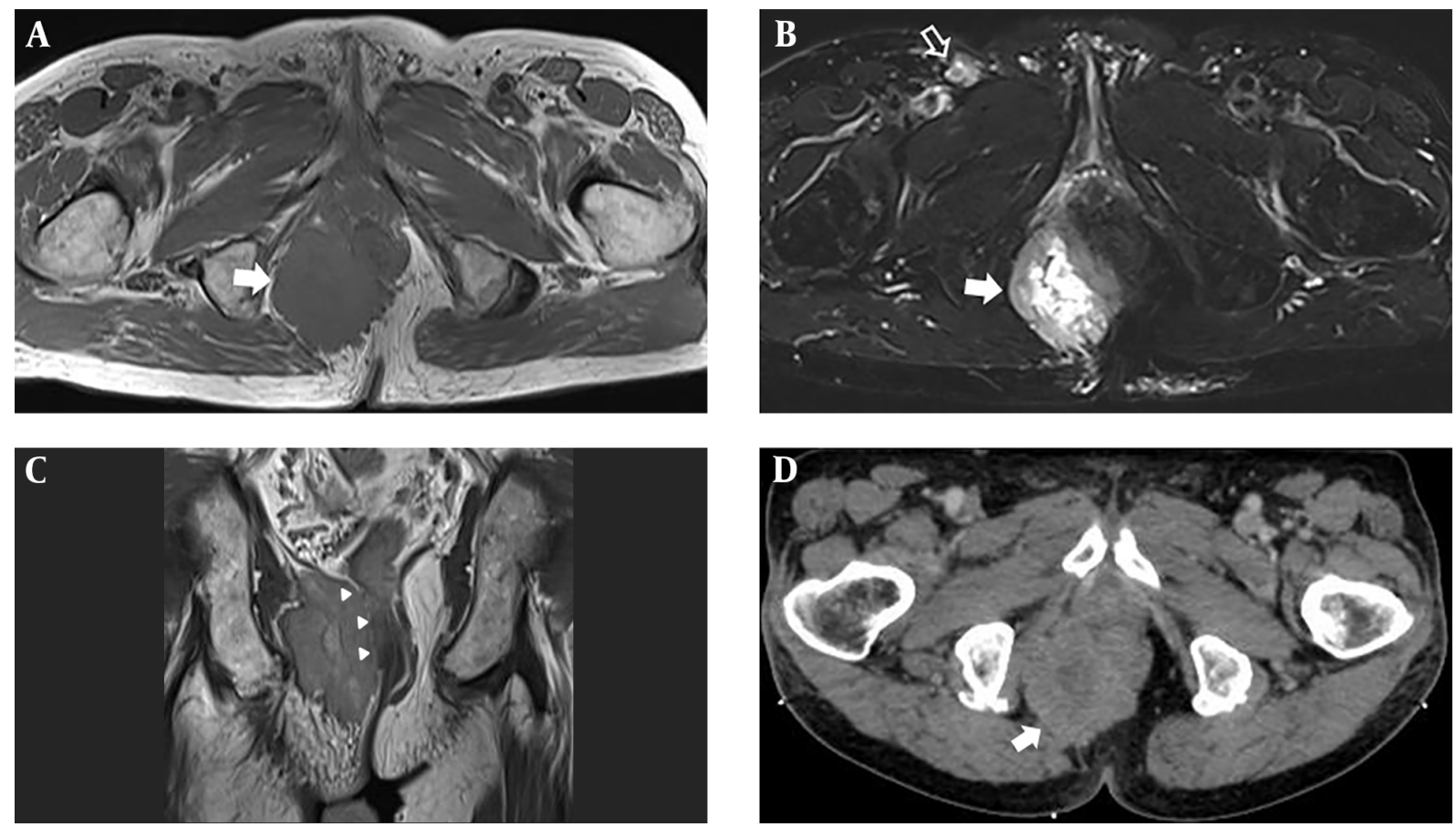

Figure 1. Metastatic tumor in the right perianal area. A, Axial T1-weighted image shows a mass with intermediate signal intensity (arrow); B, Axial fat suppressed T2-weighted half-Fourier single-shot turbo spin-echo (HASTE) image shows a central high signal intensity and peripheral intermediate signal intensity lesion (arrow). Note the enlarged right inguinal lymph node (empty arrow); C, Coronal proton-weighted image displays a large mass at the right ischiorectal/ischioanal fossa with invasion of the right external anal sphincter, puborectal, and levator ani muscles (arrowheads), and suspicious invasion of the internal anal sphincter; D, Contrast-enhanced axial CT image shows an irregularly shaped, heterogeneous enhancing mass in the right perianal area (arrow).

scan revealed partial response based on RECIST 1.1 criteria with a significant decrease in the size of the perianal metastasis $(2.8 \times 2.7 \times 1.9 \mathrm{~cm})$. However, an enlargement in the right inguinal lymph node was observed $(4 \times 6.3 \times 4.2 \mathrm{~cm}$, image not shown).

\section{Discussion}

SCLC is the most common primary pulmonary neuroendocrine malignancy, and the most common sites of metastases are the bones, liver, adrenal glands, and brain. Gastrointestinal metastases of primary lung cancer have been reported to have an incidence ranging from 4.7 to $14 \%$ on autopsy, and the small bowel is the most commonly reported site $(1,2)$. Perianal/anal or perirectal/rectal metastasis originating from primary lung cancer is extremely rare, with only 15 published reports (1-8). To the best of our knowledge, there are only three reports of perianal or perirectal metastases from SCLC, including the present study (6-8).

The symptoms of perianal/perirectal metastases may be nonspecific or similar to those of primary gastrointestinal malignancies $(5,6)$. Diagnosis may be delayed since anal cancers are generally attributed to hemorrhoids (9).
In previous studies that analyzed cases of perianal and anal metastases from primary lung cancers, abscess formation was the most prevalent presentation (1). In the present case, the patient experienced perianal pain, and physical examination showed an internal hemorrhoid, substantiating the possibility of misdiagnosis. In the diagnosis of anal cancer or metastasis, standard workup includes digital rectal examination and systemic radiological evaluation (9). The recommendations published by the European Society for Medical Oncology in 2014 advocate the use of pelvic MRI or endoanal ultrasound for diagnosis (9). CT can be used to assess the extent of distant metastases, mainly in the liver and lungs. In the present study, enhanced CT imaging revealed a heterogeneous enhancement of the perianal metastatic tumor from the lung, with a necrotic portion and enlarged inguinal lymph nodes. The mass showed intermediate signal intensity on T1-weighted imaging and intermediate to high signal intensity with a necrotic portion on T2-weighted imaging. Suto et al. have reported that muscle metastasis showed slightly low signal intensity on T1-weighted imaging but equal or higher signal intensity on T2-weighted imaging compared to the surrounding muscle and a heterogeneous contrast enhancement on contrast-enhanced T1-weighted imaging (10). In 

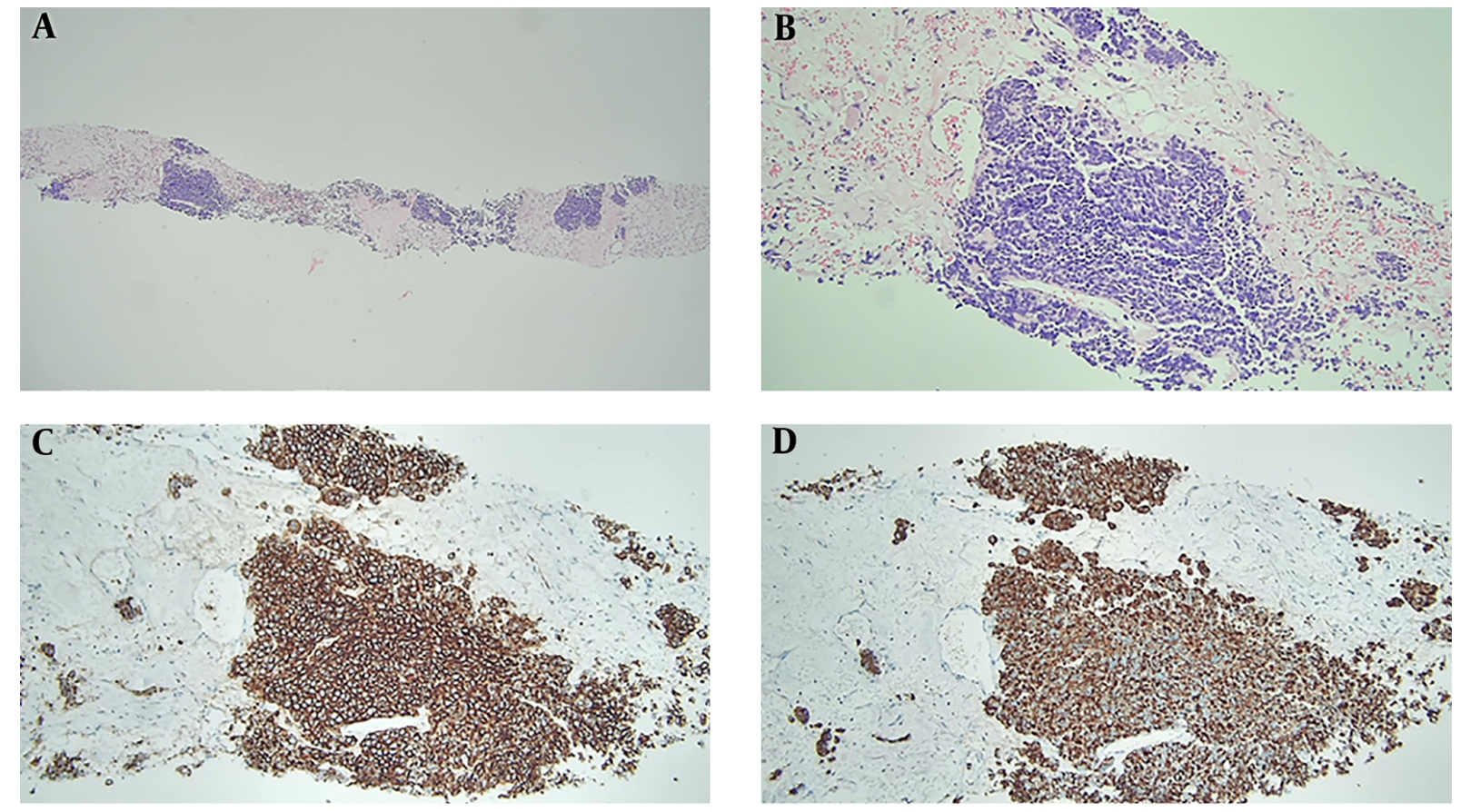

Figure 2. Histopathological features of perianal metastasis from small cell lung carcinoma. A, Specimen from the perianus is composed of nests of tumor cells with hyalinized stroma (hematoxylin and eosin, $\times 40$ ); B, Tumor cells show fine chromatin with nuclear molding (hematoxylin and eosin, $\times 200$ ); C and D, Tumor cells are positive for CD56 (C) and cytokeratin (D) on immunohistochemical staining $(\times 200)$.

the present case, the appearance of perianal metastasis was nonspecific on MRI, but MRI might define the extent of the lesion better.

For treatment, patients should undergo systemic single chemotherapy or combined chemotherapy and radiation therapy (1). However, patients with perianal or perirectal malignancies may present with intestinal stenosis or obstruction, which requires urgent surgical intervention. Palliative surgery can also be performed in cases where hemorrhage and external mass cause severe discomfort to the patient (1). Pulmonary malignancy with gastrointestinal/colonic metastasis has been reported to have a poor prognosis, with a median survival time of 3 months (4). In the present case, radiation therapy was performed on the perianal area without surgical intervention since the patient had already been receiving systemic chemotherapy for SCLC.

In conclusion, our report describes a rare case of perianal metastasis from SCLC. To the best of our knowledge, this is the first report on perianal metastasis from SCLC diagnosed using MRI. Furthermore, spread of lung cancer to atypical areas should not be overlooked, and radiologists and clinicians should be aware of its imaging features.

\section{Footnotes}

Authors' Contributions: Conceptualization, Yun Sun Choi and Won Jae Choi; Data curation, all authors; Formal analysis, Yun Sun Choi, Yoon Young Jung, and Won Jae Choi; Investigation, all authors; Methodology, all authors; Project administration, Yun Sun Choi; Resources, all authors; Supervision, Yun Sun Choi; Validation, Yun Sun Choi and Won Jae Choi; Visualization, Won Jae Choi and Yun Sun Choi; Writing - original draft, Won Jae Choi and Yun Sun Choi; Writing - review and editing, Yun Sun Choi, Yoon Young Jung, and Won Jae Choi; Approval of final manuscript, all authors.

Conflict of Interests: None declared.

Ethical Approval: None declared.

Funding/Support: None declared.

Informed Consent: Authors have received an informed consent from the patient.

\section{References}

1. Al-Tarakji M, Feilchenfeldt J, Haidar A, Szabados L, Abdelaziem S, Sayed A, et al. Rare occurrence of metastasis from lung cancer to the anus: Case report and review of the literature. World J Surg Oncol. 2016;14(1):157. doi: 10.1186/s12957-016-0909-2. [PubMed: 27278524]. [PubMed Central: PMC4898473]. 
2. Dhandapani RG, Anosike C, Ganguly A. Non-small cell lung carcinoma metastasis to the anus. BMJ Case Rep. 2016;2016. doi: 10.1136/bcr-2016214653. [PubMed: 27130556]. [PubMed Central: PMC4854120].

3. Cedres S, Mulet-Margalef N, Montero MA, Martinez P, Martinez A, Felip E. Rectal metastases from squamous cell carcinoma: A case report and review of the literature. Case Rep Med. 2012;2012:947524. doi: 10.1155/2012/947524. [PubMed: 22567021]. [PubMed Central: PMC3332391]

4. Noergaard MM, Stamp IM, Bodtger U. Disseminated lung cancer presenting as a rectal mass. Eur Clin Respir J. 2016;3:31726. doi: 10.3402/ecrj.v3.31726. [PubMed: 27683028]. [PubMed Central: PMC5040824].

5. Imai $\mathrm{H}$, Inafuku $\mathrm{K}$, Wakuda $\mathrm{K}$, Ono $\mathrm{A}$, Taira $\mathrm{T}$, Kenmotsu $\mathrm{H}$, et al. Perianal metastasis of non-small cell lung cancer. Intern Med. 2014;53(11):1149-52. doi: 10.2169/internalmedicine.53.1155. [PubMed: 24881739].

6. Guerra F, Amore Bonapasta S, Tumbiolo S, Gentile E, Coratti A. Lung cancer metastasis to the external anal sphincter mimicking perianal abscess. Int J Colorectal Dis. 2015;30(4):581-2. doi: 10.1007/s00384-0142032-6. [PubMed: 25316576].

7. Kawahara K, Akamine S, Takahashi T, Nakamura A, Kusano H, Nakagoe T, et al. Anal metastasis from carcinoma of the lung: Report of a case. Surg Today.1994;24(12):1101-3. doi:10.1007/BF01367465. [PubMed: 7780236].

8. Johnson AO, Allen MB. Rectal metastases from small cell lung cancer. Respir Med. 1995;89(3):223-5. doi: 10.1016/0954-6111(95)90252-X. PubMed: 7746917].

9. Durot C, Dohan A, Boudiaf M, Servois V, Soyer P, Hoeffel C. Cancer of the anal canal: Diagnosis, staging and follow-up with MRI. Korean J Radiol. 2017;18(6):946-56. doi: 10.3348/kjr.2017.18.6.946. [PubMed: 29089827]. [PubMed Central: PMC5639160].

10. Suto Y, Yamaguchi Y, Sugihara S. Skeletal muscle metastasis from lung carcinoma: MR findings. J Comput Assist Tomogr. 1997;21(2):304-5. doi: 10.1097/00004728-199703000-00027. [PubMed: 9071306]. 